\title{
The unconditional case of the complex $S$-inequality
}

\author{
Piotr Nayar ${ }^{*}$ Tomasz Tkocz ${ }^{\dagger}$
}

\begin{abstract}
In this note we prove the complex counterpart of the S-inequality for complete Reinhardt sets. In particular, this result implies that the complex S-inequality holds for unconditional convex sets.
\end{abstract}

2010 Mathematics Subject Classification. Primary 60G15; Secondary $60 \mathrm{E} 15$.

Key words and phrases. S-inequality, Gaussian measure, Complete Reinhardt set, Unconditional complex norm, Entropy.

\section{Introduction}

Studying various aspects of a Gaussian measure in a Banach space one often needs precise estimates on measures of balls and their dilations. This gives raise to the question how the function $(0, \infty) \ni t \mapsto \mu(t B)$ behaves. Here $B$ is a convex and symmetric subset of some Banach space, i.e. an unit ball with respect to some norm, and $\mu$ is a Gaussian measure. Thanks to certain approximation arguments we may only deal with the simplest spaces, namely $\mathbb{R}^{n}$ or $\mathbb{C}^{n}$. In the former case the issue is well understood due to R. Latała and K. Oleszkiewicz. Denote by $\gamma_{n}$ the standard Gaussian measure on $\mathbb{R}^{n}$, i.e. the measure with the density at a point $\left(x_{1}, \ldots, x_{n}\right)$ equal to $\frac{1}{\sqrt{2 \pi}^{n}} \exp \left(-x_{1}^{2} / 2-\ldots-x_{n}^{2} / 2\right)$. In LO1 it is shown that for a symmetric convex body $K \subset \mathbb{R}^{n}$ and the strip $P=\left\{x \in \mathbb{R}^{n}|| x_{1} \mid \leq p\right\}$, where $p$ is chosen so that $\gamma_{n}(K)=\gamma_{n}(P)$, we have

$$
\gamma_{n}(t K) \geq \gamma_{n}(t P), \quad t \geq 1
$$

This result is called S-inequality. The interested reader is also referred to a concise survey Lat.

In the present note we would like to focus on S-inequality for sets which correspond to unit balls with respect to unconditional norms on $\mathbb{C}^{n}$. Some partial results concerning general case has been recently obtained in Tko].

Definitions and preliminary statements are provided in Section 2. Section 3 is devoted to the main result. It also contains a proof of a one-dimensional inequality, which bounds entropy, and seems to be the heart of the proof of our main theorem.

\footnotetext{
*Research partially supported by NCN Grant no. 2011/01/N/ST1/01839.

${ }^{\dagger}$ Research partially supported by NCN Grant no. 2011/01/N/ST1/05960.
} 


\section{Preliminaries}

We define the standard Gaussian measure $\nu_{n}$ on the space $\mathbb{C}^{n}$ via the formula

$$
\nu_{n}(A)=\gamma_{2 n}(\tau(A)), \quad \text { for any Borel set } A \subset \mathbb{C}^{n},
$$

where $\mathbb{C}^{n} \stackrel{\tau}{\longmapsto} \mathbb{R}^{2 n}$ is the bijection given by

$$
\tau\left(z_{1}, \ldots, z_{n}\right)=\left(\mathfrak{R e} z_{1}, \mathfrak{I m} z_{1}, \ldots, \mathfrak{R e} z_{n}, \mathfrak{I m} z_{n}\right) .
$$

We adopt the notation $\mathbb{R}_{+}=[0,+\infty)$. Later on we will also extensively use the notion of the entropy of a function $h: X \longrightarrow \mathbb{R}_{+}$with respect to a probability measure $\mu$ on a measurable space $X$

$$
\operatorname{Ent}_{\mu} f=\int_{X} f(x) \ln f(x) \mathrm{d} \mu(x)-\left(\int_{X} f(x) \mathrm{d} \mu(x)\right) \ln \left(\int_{X} f(x) \mathrm{d} \mu(x)\right) .
$$

We say that a closed subset of $\mathbb{C}^{n}$ supports the complex $S$-inequality, $S \mathbb{C}$ inequality for short, if for any cylinder $C=\left\{z \in \mathbb{C}^{n}|| z_{1} \mid \leq R\right\}$ we have

$$
\nu_{n}(K)=\nu_{n}(C) \quad \Longrightarrow \quad \nu_{n}(t K) \geq \nu_{n}(t C), \quad \text { for } t \geq 1 \text {. }
$$

Note that the natural counterpart of $S$-inequality in the complex case is the following conjecture due to Prof. A. Pełczyński, which has already been discussed in [Tko].

Conjecture. All closed subsets $K$ of $\mathbb{C}^{n}$ which are rotationally symmetric, that is $e^{i \theta} K=K$ for any $\theta \in \mathbb{R}$, support $S \mathbb{C}$-inequality.

In the present paper we are interested in the class $\mathfrak{R}$ of all closed sets in $\mathbb{C}^{n}$ which are Reinhardt complete, i.e. along with each point $\left(z_{1}, \ldots, z_{n}\right)$ such a set contains all points $\left(w_{1}, \ldots, w_{n}\right)$ for which $\left|w_{k}\right| \leq\left|z_{k}\right|, k=1, \ldots, n$ (consult for instance the textbook [Sh, I.1.2, pp. 8-9]). The key point is that this class contains all unit balls with respect to unconditional norms on $\mathbb{C}^{n}$. Recall that a norm $\|\cdot\|$ is said to be unconditional if $\left\|\left(e^{i \theta_{1}} z_{1}, \ldots, e^{i \theta_{n}} z_{n}\right)\right\|=\|z\|$ for all $z \in \mathbb{C}^{n}$ and $\theta_{1}, \ldots, \theta_{n} \in \mathbb{R}$.

The goal is to prove that all sets from the class $\mathfrak{R}$ support $S \mathbb{C}$-inequality. Now we establish some general yet simple observations which allows us to reduce the problem to a one-dimensional entropy inequality.

Proposition 1. A closed subset $K$ of $\mathbb{C}^{n}$ supports $S \mathbb{C}$-inequality if for any cylinder $C$ we have

$$
\nu_{n}(K)=\left.\nu_{n}(C) \Longrightarrow \frac{\mathrm{d}}{\mathrm{d} t} \nu_{n}(t K)\right|_{t=1} \geq\left.\frac{\mathrm{d}}{\mathrm{d} t} \nu_{n}(t C)\right|_{t=1}
$$

The proof is essentially given in [KS, Lemma 1], so we skip it. For any closed set $A$ the derivative of the function $t \mapsto \nu_{n}(t A)$ is easy to compute. Indeed,

$$
\begin{aligned}
\left.\frac{\mathrm{d}}{\mathrm{d} t} \nu_{n}(t A)\right|_{t=1} & =\left.\frac{\mathrm{d}}{\mathrm{d} t} \int_{t A} e^{-|z|^{2} / 2} \mathrm{~d} z\right|_{t=1}=\left.\frac{\mathrm{d}}{\mathrm{d} t} \int_{A} t^{2 n} e^{-t^{2}|w|^{2} / 2} \mathrm{~d} w\right|_{t=1} \\
& =2 n \nu_{n}(A)-\int_{A}|z|^{2} \mathrm{~d} \nu_{n}(z)
\end{aligned}
$$


Moreover, the integral of $|z|^{2}$ over a cylinder $C$ may be expressed explicitly in terms of the measure $\nu_{n}(C)$. Namely,

$$
\int_{C}|z|^{2} \mathrm{~d} \nu_{n}(z)=2\left(1-\nu_{n}(C)\right) \ln \left(1-\nu_{n}(C)\right)+2 n \nu_{n}(C) .
$$

Combining these two remarks with the preceding proposition we obtain an equivalent formulation of the problem..

Proposition 2. A closed subset $K$ of $\mathbb{C}^{n}$ supports $S \mathbb{C}$-inequality if and only if

$$
\int_{K}|z|^{2} \mathrm{~d} \nu_{n}(z) \leq 2 n \nu_{n}(K)+2\left(1-\nu_{n}(K)\right) \ln \left(1-\nu_{n}(K)\right) .
$$

\section{Main result}

We aim at proving the aforementioned main result, which reads as follows

Theorem 1. Any set from the class $\mathfrak{R}$ supports $S \mathbb{C}$-inequality.

We begin with a one-dimensional entropy inequality.

Lemma 1. Let $\mu$ be a Borel probability measure on $\mathbb{R}_{+}$and suppose $f: \mathbb{R}_{+} \longrightarrow$ $\mathbb{R}_{+}$is a bounded and non-decreasing function. Then

$$
\operatorname{Ent}_{\mu} f \leq-\int_{\mathbb{R}_{+}} f(x)(1+\ln \mu((x, \infty))) \mathrm{d} \mu(x) .
$$

Proof. Using homogeneity of both sides of (5), without loss of generality, we can assume that $\int_{\mathbb{R}_{+}} f \mathrm{~d} \mu=1$. Then we may rewrite the assertion of the lemma as follows

$$
\int_{\mathbb{R}_{+}} \ln \left(f(x) \int_{(x, \infty)} \mathrm{d} \mu(t)\right) f(x) \mathrm{d} \mu(x) \leq-1 .
$$

Introduce the probability measure $\nu$ on $\mathbb{R}_{+}$with the density $f$ with respect to $\mu$. Thanks to monotonicity of $f$ we might estimate the left hand side of the last inequality by

$$
\int_{\mathbb{R}_{+}} \ln (\nu((x, \infty))) \mathrm{d} \nu(x)=-\int_{0}^{\infty} \int_{0}^{1} \frac{\mathrm{d} u}{u} \mathbf{1}_{\{u \geq \nu((x, \infty))\}}(u, x) \mathrm{d} \nu(x) .
$$

Define the function

$$
H(y):=\inf \{t \mid \nu((t, \infty)) \leq y\},
$$

which is the inverse tail function, and observe that

$$
\{(u, x) \mid u \geq \nu((x, \infty))\} \supset\{(u, x) \mid H(u) \leq x\},
$$

as $u \geq \nu((H(u), \infty)) \geq T(x)$. This leads to

$$
\begin{aligned}
-\int_{0}^{\infty} \int_{0}^{1} \frac{\mathrm{d} u}{u} \mathbf{1}_{\{u \geq \nu((x, \infty))\}}(u, x) \mathrm{d} \nu(x) & \leq-\int_{0}^{\infty} \int_{0}^{1} \frac{\mathrm{d} u}{u} 1_{\{H(u) \leq x\}}(u, x) \mathrm{d} \nu(x) \\
& =-\int_{0}^{1} \nu([H(u), \infty)) \frac{\mathrm{d} u}{u} .
\end{aligned}
$$

Since $u \leq \nu([H(u), \infty))$, we finally get the desired estimation. 
Now, for a certain class of functions, we establish the multidimensional version of inequality (5). For the simplicity, we formulate this result for the Gaussian measure.

Lemma 2. Let $g: \mathbb{C}^{n} \longrightarrow \mathbb{R}_{+}$be a bounded function satisfying

1) $g\left(\left(e^{i \theta_{1}} z_{1}, \ldots, e^{i \theta_{n}} z_{n}\right)\right)=g(z)$ for any $z \in \mathbb{C}^{n}$ and $\theta_{1}, \ldots, \theta_{n} \in \mathbb{R}$,

2) for any $w, z \in \mathbb{C}^{n}$ the condition $\left|w_{k}\right| \leq\left|z_{k}\right|, k=1, \ldots, n$ implies $g(w) \leq g(z)$.

Then

$$
\operatorname{Ent}_{\nu_{n}} g \leq \int_{\mathbb{C}^{n}} g(z)\left(\frac{|z|^{2}}{2}-n\right) \mathrm{d} \nu_{n}(z)
$$

Proof. One piece of notation: for a fixed vector $r=\left(r_{1}, \ldots, r_{n}\right) \in\left(\mathbb{R}_{+}\right)^{n}$ we denote $r^{k}=\left(r_{1}, \ldots, r_{k-1}, r_{k+1}, \ldots, r_{n}\right) \in\left(\mathbb{R}_{+}\right)^{n-1}$, and then define the functions

$$
g_{k}^{r^{k}}(x)=g\left(r_{1}, \ldots, r_{k-1}, x, r_{k+1}, \ldots, r_{n}\right), \quad k=1, \ldots, n .
$$

Notice that for a function $h: \mathbb{C} \longrightarrow \mathbb{R}_{+}$obeying the property 1) we get

$$
\int_{\mathbb{C}} h(z) \mathrm{d} \nu_{1}(z)=\frac{1}{2 \pi} \int_{0}^{2 \pi} \int_{0}^{\infty} h\left(r e^{i \theta}\right) e^{-r^{2} / 2} r \mathrm{~d} r \mathrm{~d} \theta=\int_{0}^{\infty} h(r) \mathrm{d} \mu(r),
$$

where $\mu$ denotes the probability measure on $\mathbb{R}_{+}$with the density at $r$ given by $r e^{-r^{2} / 2}$. Therefore

$$
\begin{aligned}
\int_{\mathbb{C}^{n}} g(z)\left(\frac{|z|^{2}}{2}-n\right) \mathrm{d} \nu_{n}(z) & =\int_{\left(\mathbb{R}_{+}\right)^{n}} g(r)\left(\frac{\sum_{k=1}^{n} r_{k}^{2}}{2}-n\right) \mathrm{d} \mu^{\otimes n}(r) \\
& =\int_{\left(\mathbb{R}_{+}\right)^{n}} \sum_{k=1}^{n}\left[\int_{\mathbb{R}_{+}} g_{j}^{r^{j}}(x)\left(\frac{x^{2}}{2}-1\right) \mathrm{d} \mu(x)\right] \mathrm{d} \mu^{\otimes n}(r) .
\end{aligned}
$$

Applying Lemma 1 for the function $g_{j}^{r^{j}}$ and the measure $\mu$ we obtain the estimation

$$
\begin{aligned}
\int_{\mathbb{C}^{n}} g(z)\left(\frac{|z|^{2}}{2}-n\right) \mathrm{d} \nu_{n}(z) & \geq \int_{\left(\mathbb{R}_{+}\right)^{n}} \sum_{k=1}^{n} \operatorname{Ent}_{\mu} g_{j}^{r^{j}} \mathrm{~d} \mu{ }^{\otimes n}(r) \\
& \geq \operatorname{Ent}_{\mu} \otimes n \\
& =\operatorname{Ent}_{\nu_{n}} g
\end{aligned}
$$

where the last inequality follows from subadditivity of entropy (for example see [Led, Proposition 5.6]).

Proof of Theorem [1. Fix $K \in \mathfrak{R}$. In order to show (4) we introduce the function $g(z)=1-\mathbf{1}_{K}(z)$. We adopt the standard convention that $0 \ln 0=0$, hence the desired inequality is equivalent to (6). Thus the application of Lemma 2 for the function $g$ finishes the proof.

Theorem 1 immediately implies that the Cartesian products of cylinders support $S \mathbb{C}$-inequality. As a consequence, $S \mathbb{C}$-inequality possesses a tensorization property.

Corollary 1. Assume sets $K_{1} \subset \mathbb{C}^{n_{1}}, \ldots, K_{\ell} \subset \mathbb{C}^{n_{\ell}}$ support $S \mathbb{C}$-inequality. Then the set $K_{1} \times \ldots \times K_{\ell}$ also supports $S \mathbb{C}$-inequality. 


\section{Acknowledgements}

We would like to thank R. Adamczak for his remark regarding Lemma 1, which led to the present general formulation.

The work was done while the second named author was participating in The Kupcinet-Getz International Summer Science School at the Weizmann Institute of Science in Rehovot, Israel.

\section{A $S$-inequality for the exponential measure in the unconditional case}

Let $\lambda$ be the symmetric exponential measure on $\mathbb{R}$, i.e.

$$
\mathrm{d} \lambda(x)=\frac{1}{2} e^{-|x|} \mathrm{d} x, \quad x \in \mathbb{R},
$$

and let $\lambda_{n}=\lambda \otimes \ldots \otimes \lambda$ be the standard exponential measure on $\mathbb{R}^{n}$, i.e.

$$
\mathrm{d} \lambda(x)=\frac{1}{2^{n}} e^{-|x|_{1}} \mathrm{~d} x, \quad x \in \mathbb{R}^{n},
$$

where we denote $\left|\left(x_{1}, \ldots, x_{n}\right)\right|_{1}=\sum_{i=1}^{n}\left|x_{i}\right|$. It has been recently noticed that the technique of the paper applies also to the $S$-inequality for the measure $\lambda_{n}$. The result reads as follows

Theorem 2. For any closed convex subset $K \subset \mathbb{R}^{n}$ which is unconditional, i.e. $\left(\epsilon_{1} x_{1}, \ldots, \epsilon_{n} x_{n}\right) \in K$ whenever $\left(x_{1}, \ldots, x_{n}\right) \in K$ and $\epsilon_{1}, \ldots, \epsilon_{n} \in\{-1,1\}$, and for any strip $P=\left\{x \in \mathbb{R}^{n}|| x_{1} \mid \leq p\right\}, p \geq 0$, we have

$$
\lambda_{n}(K)=\lambda_{n}(P) \quad \Longrightarrow \quad \forall t \geq 1 \lambda_{n}(t K) \geq \lambda_{n}(t P),
$$

and, equivalently,

$$
\lambda_{n}(K)=\lambda_{n}(P) \quad \Longrightarrow \quad \forall t \leq 1 \lambda_{n}(t K) \leq \lambda_{n}(t P) .
$$

Proof. The equivalence between (77) and (8) is straightforward. For instance, assume the latter does not hold. Then, there is $t_{0}<1$ such that $\lambda_{n}\left(t_{0} K\right)>$ $\lambda_{n}\left(t_{0} P\right)$. So, we can find $s_{0}<1$ for which $\lambda_{n}\left(s_{0} t_{0} K\right)=\lambda_{n}\left(s_{0} t_{0} P\right)$. Using (7) we get a contradiction

$$
\lambda_{n}(K)>\lambda_{n}\left(s_{0} K\right)=\lambda_{n}\left(\frac{1}{t_{0}}\left(s_{0} t_{0} K\right)\right) \geq \lambda_{n}\left(\frac{1}{t_{0}}\left(t_{0} P\right)\right)=\lambda_{n}(P)=\lambda_{n}(K) .
$$

We essentially follow the proof of Theorem 1. Hence, first of all, as in Proposition 1] we notice that we might equivalently prove that

$$
\lambda_{n}(K)=\left.\lambda_{n}(P) \Longrightarrow \frac{\mathrm{d}}{\mathrm{d} t} \lambda_{n}(t K)\right|_{t=1} \geq\left.\frac{\mathrm{d}}{\mathrm{d} t} \lambda_{n}(t C)\right|_{t=1} .
$$

Again, a trivial computation shows that

$$
\left.\frac{\mathrm{d}}{\mathrm{d} t} \lambda_{n}(t K)\right|_{t=1}=n \lambda_{n}(K)-\int_{K}|x|_{1} \mathrm{~d} \lambda_{n}(x) .
$$


Thus, we would like to prove that

$$
\lambda_{n}(K)=\lambda_{n}(P) \quad \Longrightarrow \quad \int_{K}|x|_{1} \mathrm{~d} \lambda_{n}(x) \leq \int_{P}|x|_{1} \mathrm{~d} \lambda_{n}(x) .
$$

One another easy computation yields

$$
\int_{P}|x|_{1} \mathrm{~d} \lambda_{n}(x)=n\left(1-e^{-p}\right)-p e^{-p} .
$$

But $\lambda_{n}(K)=\lambda_{n}(P)=1-e^{-p}$, so we get

$$
\int_{P}|x|_{1} \mathrm{~d} \lambda_{n}(x)=n-n \lambda_{n}\left(K^{\prime}\right)+\lambda_{n}\left(K^{\prime}\right) \ln \lambda_{n}\left(K^{\prime}\right),
$$

where $K^{\prime}=\mathbb{R}^{n} \backslash K$. Since $\int_{\mathbb{R}^{n}}|x|_{1} \mathrm{~d} \lambda_{n}(x)=n$, (11) is equivalent to

$$
-\lambda_{n}\left(K^{\prime}\right) \ln \lambda_{n}\left(K^{\prime}\right) \leq \int_{K^{\prime}}\left(|x|_{1}-n\right) \mathrm{d} \lambda_{n}(x) .
$$

Now we introduce the function $g(x)=\mathbf{1}_{K^{\prime}}(x)$. Then the above inequality may be rewritten to

$$
\operatorname{Ent}_{\lambda_{n}} g \leq \int_{\mathbb{R}^{n}} g(x)\left(|x|_{1}-n\right) \mathrm{d} \lambda_{n}(x) .
$$

Thanks to unconditionality of $K$ the function $g$ is even with respect to each coordinate. Using in addition convexity, we check that it is nondecreasing with respect to each coordinate. These properties as well as certain one-dimensional inequality, which is deduced from Lemma 1 for the measure with the density $e^{-x}$ on $\mathbb{R}_{+}$, allow us to prove inequality (12) in the same manner as in Lemma 2.

Following the method of [LO1, Corollary 3] we obtain the result concerning the comparison of moments.

Corollary 2. Let $\|\cdot\|$ be a norm on $\mathbb{R}^{n}$ which is unconditional, i.e.

$$
\mid\left(\epsilon_{1} x_{1}, \ldots, \epsilon_{n} x_{n}\right)\|=\|\left(x_{1}, \ldots, x_{n}\right) \|,
$$

for any $x_{j} \in \mathbb{R}$ and $\epsilon_{j} \in\{-1,1\}$. Then for $p \geq q>0$

$$
\left(\int_{\mathbb{R}^{n}}\|x\|^{p} \mathrm{~d} \lambda_{n}(x)\right)^{1 / p} \leq C_{p, q}\left(\int_{\mathbb{R}^{n}}\|x\|^{q} \mathrm{~d} \lambda_{n}(x)\right)^{1 / q},
$$

where the constant $C_{p, q}=\left(\int_{\mathbb{R}}|x|^{p} \mathrm{~d} \lambda(x)\right)^{1 / p} /\left(\int_{\mathbb{R}}|x|^{q} \mathrm{~d} \lambda(x)\right)^{1 / q}$ is the best possible.

Proof. The proof hinges on the fact that a ball $K=\left\{x \in \mathbb{R}^{n}\|x\| \leq t\right\}$ with respect to the norm $\|\cdot\|$ is a closed convex unconditional set, so that Theorem 2 can be applied.

Remark 1. It has been tempting to see how the proof might have worked for product measures with the density $C_{\rho}^{n} e^{-\sum_{i=1}^{n} \rho\left(\left|x_{i}\right|\right)}$, where $\rho$ is, e.g., positive convex and increasing function on $(0, \infty)$ and $\rho(0)=0$. The only problem is that this in not entropy but another functional, constructed out of $\rho$, that appears in (12). In the case of functions $\rho(x)=x^{p}, p>1$, we did check that Corollary 3 of LO2 (consult there Definition 4 as well) does not give that such functionals possess desired properties such as subadditivity. From this point of view the exponential measure seems to be quite exceptional. 


\section{References}

[KS] S. Kwapień, J. Sawa, On some conjecture concerning Gaussian measures of dilatations of convex symmetric sets, Studia Math., 105 (1993), no. 2, 173-187. MR1226627 (94g:60011)

[Lat] R. Latała, On some inequalities for Gaussian measures (English summary), Proceedings of the International Congress of Mathematicians, Vol. II (Beijing, 2002), 813-822, Higher Ed. Press, Beijing, 2002. MR1957087 (2004b:60055)

[LO1] R. Latała, K. Oleszkiewicz, Gaussian measures of dilatations of convex symmetric sets, Ann. Probab. 27 (1999), no. 4, 1922-1938. MR1742894 (2000k:60062)

[LO2] R. Latała, K. Oleszkiewicz, Between Sobolev and Poincaré, Geometric aspects of functional analysis, 147-168, Lecture Notes in Math., 1745, Springer, Berlin, 2000. MR1796718 (2002b:60025)

[Led] M. Ledoux, The concentration of measure phenomenon. Mathematical Surveys and Monographs, 89. American Mathematical Society, Providence, RI, 2001. MR1849347 (2003k:28019)

[Sh] B. V. Shabat, Introduction to complex analysis. Part II. Functions of several variables. Translated from the third (1985) Russian edition by J. S. Joel. Translations of Mathematical Monographs, 110. American Mathematical Society, Providence, RI, 1992. MR1192135 (93g:32001)

[Tko] T. Tkocz, Gaussian measures of dilations of convex rotationally symmetric sets in $\mathbb{C}^{n}$, Electron. Commun. Probab. 16 (2011), 38-49. MR2763527 (Review)

Piotr Nayar

Institute of Mathematics, University of Warsaw,

Banacha 2,

02-097 Warszawa, Poland.

nayar@mimuw.edu.pl

Tomasz Tkocz

Institute of Mathematics, University of Warsaw,

Banacha 2,

02-097 Warszawa, Poland.

tkocz@mimuw.edu.pl 